\title{
Modeling and Optimization of Inventory-Distribution Routing Problem for Agriculture Products Supply Chain
}

\author{
Li Liao, ${ }^{1,2}$ Jianfeng $\mathrm{Li}^{2}$ and Yaohua $\mathrm{Wu}^{1}$ \\ ${ }^{1}$ School of Control Science and Engineering, Shandong University, Jinan 250061, China \\ ${ }^{2}$ School of Mechanical Engineering, Shandong University, Jinan 250061, China \\ Correspondence should be addressed to Li Liao; liaoli@sdu.edu.cn
}

Received 9 November 2012; Accepted 24 January 2013

Academic Editor: Xiang Li

Copyright (c) $2013 \mathrm{Li}$ Liao et al. This is an open access article distributed under the Creative Commons Attribution License, which permits unrestricted use, distribution, and reproduction in any medium, provided the original work is properly cited.

\begin{abstract}
Mathematical models of inventory-distribution routing problem for two-echelon agriculture products distribution network are established, which are based on two management modes, franchise chain and regular chain, one-to-many, interval periodic order, demand depending on inventory, deteriorating treatment cost of agriculture products, start-up costs of vehicles and so forth. Then, a heuristic adaptive genetic algorithm is presented for the model of franchise chain. For the regular chain model, a two-layer genetic algorithm based on oddment modification is proposed, in which the upper layer is to determine the distribution period and quantity and the lower layer is to seek the optimal order cycle, quantity, distribution routes, and the rational oddment modification number for the distributor. By simulation experiments, the validity of the algorithms is demonstrated, and the two management modes are compared.
\end{abstract}

\section{Introduction}

Agriculture products supply chain management is important for reducing circulation costs and improving supply chain efficiency. A key factor in agricultural products supply chain management is inventory-distribution routing problem. Federgruen and Zipkin [1] established an inventory-transportation integrated optimization model based on one-warehouse and multiretailer distribution network, and Bender's decomposition approach is used. Burns et al. [2] discussed the minimum cost problem on joint optimization of inventory and transportation based on onedispatching point and multireceive point network under certain demand. Daganzo [3] researched the many-to-many inventory-transportation integrated optimization problem with bulk stations. Anily and Federgruen [4] studied the inventory and vehicle routing problem based on single product, certain demand, and continuous time. Trudeau and Dror [5] considered the situation about stochastic demand and the limited capacity of the vehicles. Ernst and Pyke [6] designed a heuristic algorithm to confirm the optimal delivery frequency, according to the inventorytransportation integrated optimization problem based on single distributor-single retailer under customer's random demand. Herer and Roundy [7], Viswanathan and Mathur [8], Bertazzi et al. [9], and Chen and Zheng [10] researched an inventory-transportation integrated optimization model of one-warehouse multiretailer distribution network based on heuristic algorithm to minimize the costs of inventory, ordering, and transportation. Bertazzi and Speranza [11] proposed an efficient heuristic algorithm for multilayer inventorydistribution system with certain demand, and single supplier, no shortage, single delivery vehicle with limited capacity. Wang et al. [12] designed a heuristic algorithm according to the two-echelon distribution system which takes cyclical replenishment strategy, and the terminal customer demand is uncertain. Zhao et al. [13] proposed a decomposition heuristic algorithm based on Markov decision processes and Modified $C-W$ saving algorithm under random demand. Tang Jiafu researched delivery planning problem in distribution center with the strategy of vehicle outsourcing model and multiple direct transport; an integer programming model was 
presented, and a genetic algorithm is designed to solve this model.

So far, the researches about joint optimization of inventory and transportation focus on the industrial products, without considering the metamorphism of agricultural products. Furthermore, considering that chain operation has become the main way of distribution network operation, in which the distributor makes the unified supply for the retailer. At present, there are two models about chain operation: one is regular chain mode. The distributors and the detailers belong to the same enterprise, which makes the direct operation and centralized management. The other is the franchise chain mode, on which the distributors and the detailers do not belong to the same enterprise, and they conclude management relationship through contracts. In this paper, mathematical models are established about the routing problem based on the two modes, and corresponding algorithm is designed to give some analysis and comparison.

\section{Assumptions and Notation}

A two-echelon agriculture products supply chain system which consists of one distributor and multiple retailers is considered, in which the distributor has a single supplier and delivers agriculture products to the retailers. Both supplier and retailers adapt equal periodic order strategy and order at the beginning of period. For average profit maximization of supply chain, the optimal order time, quantity, and vehicle routing for distribution are to be determined.

The mathematical model is developed based on the following assumptions and notation.

Superscript and Subscript. Subscript 0 denotes the distributor. Subscripts $i, j$ denote serial number of retailers. Superscripts $r, m$ denote serial number of order interval period and delivery vehicles, respectively.

\section{Constant and Set}

$V, M$ : denote, respectively, set of locations of distributor and retailers and set of delivery vehicles.

$N, \Delta T, n_{\max }$, and $G$ : denote, respectively, total number of retailers, minimum order interval period, maximum order cycle length of the retailer, and maximum capacity of the delivery vehicles.

$w$ : denotes wholesale price per unit of the retailers' order on the franchise chain mode.

$\theta, b$ : denote, respectively, the constant deterioration rate of agriculture products and deteriorating treatment cost per unit of deteriorating items.

$F_{0}, F_{j}$ : denote, respectively, constant order cost of distributor and retailers for each time.

$h_{0}, h$ : denote, respectively, inventory holding cost per unit per unit time of distributor and retailers.

$s_{j}, v$ : denote, respectively, inventory shortage cost and salvage value of surplus item per unit.
$B_{j}, \alpha_{j}$ : denote, respectively, the customer's basic demand, demand, and inventory correlation coefficient for retailer $j$.

$w_{0}, p$ : denote, respectively, wholesale price per unit of the distributor's order and retail price per unit of customers' order.

$d_{i j}, c_{i j}$ : denote, respectively, the minimum distance and single vehicle freight between retailer $i$ and retailer $j, i, j \in V$.

$c, c_{0}, c^{\prime}$ : denote, respectively, single vehicle freight per distance unit between different retailers, startup costs of single vehicle, and freight per unit of the distributor's order.

\section{Variables and Symbols}

$n_{j}, Q_{j}$ : denote, respectively, order period's length and order quantity of retailer $j$.

$H, Q$ : denote, respectively, order period's length and order quantity of the distributor.

$\Omega$ : distribution route, $\Omega=\left\{x_{i j}^{r m} \mid i, j \in V, m \in M, r=\right.$ $1,2, \ldots, L\}$, where $x_{i j}^{r m}$ is the adjacency relationship of single vehicle $m$ between retailer $i$ and retailer $j, x_{i j}^{r m} \in$ $\{0,1\} ; L$ is the length of distribution cycle.

$\delta_{j}^{r}$ : order symbol of retailer $j$ in the periodic interval $r, \delta_{j}^{r} \in\{0,1\}$.

$t_{j}, T_{j}$ : denote, respectively, the retailer $j$ 's time of the inventory level decreased to zero and retailer $j$ 's order cycle.

$k, L$ : denote, respectively, the distributor's order cycle coefficient and the length of delivery cycle.

$D_{j}(t), I_{j}(t)$ : denote, respectively, the customers' demand and inventory level of retailer $j$ at time $t$.

$\pi, \pi_{j}$ : denote, respectively, the distributor's profit and retailer $j$ 's profit.

$\bar{\pi}, \bar{\pi}_{j}$ : denote, respectively, the distributor's average profit and retailer $j$ 's average profit.

$\Pi$ : average profit of supple chain system.

Assumption. (1) The retailers' demand is independent, and the demand rate is related to inventory level. Padmanabhan and Vrat's [14] demand model is adopted; at any time $t$, demand of the retailer $j$ 's customer, $D_{j}(t)$, is $D_{j}(t)=$ $B_{j}+\alpha_{j} I_{j}(t)$, where $\alpha_{j}$ is demand and inventory correlation coefficient, $\alpha_{j}>0, B_{j}$, is basic demand, $B_{j} \geq 0$. Without loss of generality, profit is minus when $B_{j}=0$, that is, the retailer cannot gain profit without customers' basic demand.

(2) Considering the metamorphism of agricultural products, the longest length of retailers' order cycle is $n_{\max }$. At the ending of every order cycle, the surplus inventory is treatment at salvage value $v, v<w_{0}$.

(3) Agriculture products inventory of retailers deteriorates at constant deterioration rate $\theta, 0<\theta<1$. The 
deteriorated items can be treated in time, and the treatment cost is $b$ per deteriorated unit.

(4) Wholesale price per unit of distributor is $w_{0}$, and retailer price per unit is $p$. Inventory holding cost per unit per unit time of distributor and retailers is $h_{0}$ and $h, w_{0}+h_{0}+$ $h<p$. For franchise chain mode, wholesale price per unit of retailer's order is $w, w_{0}+h_{0}<w$. Then, $w_{0}+h_{0}+h<w+h<p$.

(5) On franchise chain mode, distributor and retailers are risk neutral, sharing information based on wholesale contract. The distributor is leader, and the retailers act as the follower. For the distributor, shortage is not allowed and bear all freight. The average profit of distributor and retailer is concave functions of order cycle.

(6) Retailers' ordering lead time is zero. Both distributor and retailers adopt equal periodic order, and the order period is integral multiple of the minimum interval period $\Delta T$. The distributor's order cycle $H$ is integral multiple of the retailers' order cycle $n_{j} \Delta T$ :

$$
H=k L \Delta T \text {, }
$$

where $k$ is integer, $k \geq 1, L$ is least common multiple of any retailer's order cycle:

$$
L=\left[n_{1}, n_{2}, \ldots, n_{N}\right],
$$

where $[\cdot, \cdot, \ldots, \cdot]$ denotes least common multiple

(7) Delivery vehicle capacity is $G$. Single vehicle transportation cost between retailer $i$ and retailer $j, c_{i j}$, is in proportion to the distance. In view of vehicle start-up costs $c_{0}$,

$$
c_{i j}=\left\{\begin{array}{ll}
c d_{i j}, & i \neq 0, \\
c_{0}+c d_{i j}, & i=0,
\end{array} \quad i \neq j, \quad \forall i, j \in V .\right.
$$

From assumptions above, the retailer's maximum inventory level equal to order quantity $Q_{j}$. Define $t_{j}$ as time of the inventory level decreased to zero; we have $d I_{j}(t) / d t=$ $-\theta I_{j}(t)-D_{j}(t)=-\left(\theta+\alpha_{j}\right) I_{j}(t)-B_{j}, t \leq t_{j}$. With the boundary condition $I\left(t_{j}\right)=0$, then we have

$$
I_{j}(t)= \begin{cases}\frac{B_{j}}{\theta+\alpha_{j}}\left[e^{\left(\theta+\alpha_{j}\right)\left(t_{j}-t\right)}-1\right], & t \leq t_{j}, \\ 0, & t>t_{j} .\end{cases}
$$

Accordingly, the retailer's maximum inventory level and order quantity are

$$
I H_{j}=Q_{j}=\left.I_{j}(t)\right|_{t=0}=\frac{B_{j}}{\theta+\alpha_{j}}\left[e^{\left(\theta+\alpha_{j}\right) t_{j}}-1\right] .
$$

Define $x_{i j}^{r m}$ as adjacent relationship in order interval period $r$ and vehicle $m$ :

$$
\begin{aligned}
& x_{i j}^{r m} \\
& = \begin{cases}1, & \text { if vehicle } m \text { directly goes to retailer } j \text { after } \\
\text { serving retailer } i \text { at the beginning of period } r & \text { otherwise, }\end{cases}
\end{aligned}
$$

$i \neq j, \forall i, j \in V, m \in M, r=1,2, \ldots, k L$.
Define $\Omega$ as distribution route:

$$
\Omega=\left\{x_{i j}^{r m} \mid i, j \in V, m \in M, r=1,2, \ldots, L\right\} .
$$

\section{Model on Franchise Chain Mode}

On franchise chain mode, supply chain is decentralized; the optimal order quantity and order cycle rely on wholesale price. The distributor delivery products according to the retailers' demand, and determine the distributor's optimal order quantity and order cycle.

\subsection{Profit Analysis on Retailor}

Proposition 1. On franchise chain mode, as far as single order cycle $n_{j} \Delta T$ of retailer $j$ is concerned, when profit of retailer $j$ is maximumzied, time of the inventory level decreased to zero, $t_{j}$, satisfies

$$
t_{j}=n_{j} \Delta T
$$

From Proposition 1, profit is maximized when $t_{j}=T_{j}$, profit of retailer $j, \pi_{j}\left(T_{j}\right)$, is

$$
\begin{aligned}
\pi_{j}\left(T_{j}\right)= & p \int_{0}^{T_{j}}\left[B_{j}+\alpha_{j} I(t)\right] d t \\
& -w \int_{0}^{T_{j}}\left[B_{j}+\left(\alpha_{j}+\theta\right) I(t)\right] d t \\
& -b \int_{0}^{T_{j}} \theta I(t) d t-h_{j} \int_{0}^{T_{j}} I(t) d t-F_{j} .
\end{aligned}
$$

The partials of (9) are in sequence of sales revenue, purchase cost, deteriorating treatment cost, inventory holding cost, constant order cost. From (4), (5), and (9), we have the average profit of retailer $j$ :

$$
\begin{aligned}
\bar{\pi}_{j}\left(T_{j}\right) & =\frac{\pi_{j}\left(T_{j}\right)}{T_{j}} \\
& =A \frac{e^{\left(\theta+\alpha_{j}\right) T_{J}}-1}{T_{j}}-\frac{F_{j}}{T_{j}}+\frac{B_{j}}{\theta+\alpha_{j}}\left[\theta(p+b)+h_{j}\right],
\end{aligned}
$$

where

$$
A=\frac{B_{j}}{\left(\theta+\alpha_{j}\right)^{2}} \cdot\left[(p-w) \alpha_{j}-(w+b) \theta-h_{j}\right] .
$$

From assumption (6), Let $d \bar{\pi}_{j}\left(T_{j}\right) / d T_{j}=0$; we have

$$
\left[1-\left(\theta+\alpha_{j}\right) T_{j}^{*}\right] e^{\left(\theta+\alpha_{j}\right) T_{j}^{*}}=1+\frac{F_{j}}{A} .
$$

Then, when profit of retailer $j$ is maximumzied, the optimal order cycle $T_{j}^{*}$ satisfies (13). According to the constant order interval $\Delta T$, we can find the optimal length of order 
cycle $n_{j}^{*}$, and $\bar{\pi}_{j}$ approach or reach the maximum value correspondingly:

$$
n_{j}^{*}=\left\{\begin{array}{l}
\left\lfloor\frac{T_{j}^{*}}{\Delta T}\right\rfloor, \\
\text { if } \bar{\pi}_{j}\left(T_{j}=\Delta T\left\lfloor\frac{T_{j}^{*}}{\Delta T}\right\rfloor\right) \geq \bar{\pi}_{j}\left(T_{j}=\Delta T\left\lceil\frac{T_{j}^{*}}{\Delta T}\right\rceil\right), \\
\left\lceil\frac{T_{j}^{*}}{\Delta T}\right\rceil, \\
\text { if } \bar{\pi}_{j}\left(T_{j}=\Delta T\left\lfloor\frac{T_{j}^{*}}{\Delta T}\right\rfloor\right)<\bar{\pi}_{j}\left(T_{j}=\Delta T\left\lceil\frac{T_{j}^{*}}{\Delta T}\right\rceil\right),
\end{array}\right.
$$

where $\lfloor\cdot\rfloor$ and $\lceil\cdot\rceil$ denote, respectively, top rounding and round down.

Correspondingly, optimal profit of retailer $j$ is

$$
\begin{aligned}
\bar{\pi}_{j}\left(n_{j}^{*} \Delta T\right)= & A \frac{e^{\left(\theta+\alpha_{j}\right) n_{j}^{*} \Delta T}-1}{n_{j}^{*} \Delta T}-\frac{F_{j}}{n_{j}^{*} \Delta T} \\
& +\frac{B_{j}}{\theta+\alpha_{j}}\left[\theta(p+b)+h_{j}\right] .
\end{aligned}
$$

3.2. Profit Analysis on Distributor. From assumption (5) and Equation (1), the distributor's order quantity $Q_{0}$ equals his retailers' total demand in one order cycle:

$$
Q_{0}=\sum_{j=1}^{N} \sum_{r=1}^{k_{0} L} \delta_{j}^{r} Q_{j}=\sum_{j=1}^{N} \sum_{l=1}^{k_{0} L / n_{j}} Q_{j}=k L \sum_{j=1}^{N} \frac{Q_{j}}{n_{j}} .
$$

Define $\delta_{j}^{r}$ as order symbol of retailer $j$ in order interval period $r$ :

$$
\delta_{j}^{r}=\left\{\begin{array}{ll}
1, & r=z n_{j}, \\
0, & r \neq z n_{j},
\end{array} \quad z\right. \text { is integer. }
$$

At the beginning of order period $r$, the distributor's delivery quantity is $Q_{j}^{r}$, so

$$
Q_{j}^{r}=\delta_{j}^{r} Q_{j}
$$

Profit of the distributor is

$$
\begin{aligned}
\pi(k, w, \Omega)= & w Q_{0}-w_{0} Q_{0}-h_{0} \sum_{j=1}^{N} \sum_{l=1}^{k L / n_{j}-1} n_{j} \Delta T Q_{j} l-c^{l} Q_{0} \\
& -\sum_{r=1}^{k L} \sum_{m \in M} \sum_{i=0}^{N} \sum_{\substack{j=0 \\
j \neq i}}^{N} c_{i j} \delta_{j}^{r} \delta_{i}^{r} x_{i j}^{r m}-F_{0} .
\end{aligned}
$$

The partials of (18) are in sequence of sales revenue, purchase cost, inventory holding cost, freight of purchase products, delivery cost, and constant order cost. From (17) and (18), we have the average profit of the distributor:

$$
\begin{aligned}
\bar{\pi}(k, w, \Omega)= & \frac{\pi(k, w, \Omega)}{k L \Delta T} \\
= & \frac{1}{\Delta T}\left[\left(w-w_{0}-c^{\prime}-\frac{h_{0}}{2} k L \Delta T\right) \sum_{j=1}^{N} \frac{1}{n_{j}} Q_{j}\right] \\
& +\frac{h_{0}}{2} \sum_{j=1}^{N} Q_{j}-\frac{1}{L \Delta T} \sum_{r=1}^{L} \sum_{m \in M} \sum_{i=0}^{N} \sum_{\substack{j=0 \\
j \neq i}}^{N} c_{i j} \delta_{j}^{r} \delta_{i}^{r} x_{i j}^{r m} \\
& -\frac{1}{k L \Delta T} F_{0} .
\end{aligned}
$$

From (19), The average profit of distributor is concave functions of $k$. We can find the optimal $k^{*}, k^{*} \geq 1$, and $\bar{\pi}$ approach or reach the maximum value correspondingly. Let $d \bar{\pi} / d k=0$, we have

$$
k= \begin{cases}1, & \lfloor\rho\rfloor \leq 1, \\ \lfloor\rho\rfloor, & \lfloor\rho\rfloor>1,\left.\bar{\pi}(k, w, \Omega)\right|_{k=\lfloor\rho\rfloor}>\left.\bar{\pi}(k, w, \Omega)\right|_{k=\lceil\rho\rceil}, \\ \lceil\rho\rceil, & \lfloor\rho\rfloor>1,\left.\bar{\pi}(k, w, \Omega)\right|_{k=\lfloor\rho\rfloor}<\left.\bar{\pi}(k, w, \Omega)\right|_{k=\lceil\rho\rceil},\end{cases}
$$

where

$$
\rho=\frac{1}{L} \sqrt{\frac{2 F_{0}}{h_{0} \Delta T \sum_{j=1}^{N}\left(1 / n_{j}\right) Q_{j}}} .
$$

3.3. Mathematical Model. On franchise chain mode, the retailers determine their order interval $n_{j} \Delta T$ and order quantity according to wholesale price $w$, then the distributor determines optimal order interval $k L \Delta T$, order quantity $Q$, and distribute route $\Omega$. The mathematical model of inventory-distribution routing problem is

$$
\begin{aligned}
& \max \bar{\pi}(k, w, \Omega) \\
&=\max _{k, w, \Omega}\left\{\frac{1}{\Delta T}\left[\left(w-w_{0}-c^{\prime}-\frac{h_{0}}{2} k L \Delta T\right) \sum_{j=1}^{N} \frac{1}{n_{j}} Q_{j}\right]\right. \\
&+\frac{h_{0}}{2} \sum_{j=1}^{N} Q_{j} \\
&\left.\quad-\frac{1}{L \Delta T} \sum_{r=1}^{L} \sum_{i=0}^{N} \sum_{j=0}^{N} c_{i j} \delta_{j}^{r} \delta_{i}^{r} x_{i j}^{r m}-\frac{1}{k L \Delta T} F\right\}
\end{aligned}
$$


s.t.

$$
\begin{aligned}
& Q_{j}=\frac{B_{j}}{\theta+\alpha_{j}}\left[e^{\left(\theta+\alpha_{j}\right) n_{j} \Delta T}-1\right], \\
& n_{j}=\left\{\begin{array}{l}
\left\lfloor\frac{T_{j}^{*}}{\Delta T}\right\rfloor, \\
\text { if } \bar{\pi}_{j}\left(T_{j}=\Delta T\left\lfloor\frac{T_{j}^{*}}{\Delta T}\right\rfloor\right) \\
\geq \bar{\pi}_{j}\left(T_{j}=\Delta T\left\lceil\frac{T_{j}^{*}}{\Delta T}\right\rceil\right),\left\lfloor\frac{T_{j}^{*}}{\Delta T}\right\rfloor \leq n_{\max }, \\
{\left[\frac{T_{j}^{*}}{\Delta T}\right\rceil,} \\
\text { if } \left.\bar{\pi}_{j}\left(T_{j}=\Delta T \mid \frac{T_{j}^{*}}{\Delta T}\right\rfloor\right) \\
<\bar{\pi}_{j}\left(T_{j}=\Delta T\left[\frac{T_{j}^{*}}{\Delta T}\right\rceil\right),\left[\frac{T_{j}^{*}}{\Delta T}\right\rceil \leq n_{\max }, \\
n_{\max }, \\
\text { else, }
\end{array}\right. \\
& \left(\theta+\alpha_{j}\right) T_{j}^{*}=1-e^{-\left(\theta+\alpha_{j}\right) T_{j}^{*}}, \\
& \bar{\pi}_{j}\left(T_{j}\right)=\frac{B_{j}}{\left(\theta+\alpha_{j}\right)^{2}} \cdot\left[(p-w) \alpha_{j}-(w+b) \theta-h_{j}\right] \\
& \times \frac{e^{\left(\theta+\alpha_{j}\right) T_{J}}-1}{T_{j}}-\frac{F_{j}}{T_{j}}+\frac{B_{j}}{\theta+\alpha_{j}}\left[\theta(p+b)+h_{j}\right] \\
& \geq 0 \text {, }
\end{aligned}
$$

$$
L=\left[n_{1}, n_{2}, \ldots, n_{N}\right],
$$

$$
k= \begin{cases}1, & \lfloor\rho\rfloor \leq 1, \\ \lfloor\rho\rfloor, & \lfloor\rho\rfloor>1,\left.\bar{\pi}(k, w, \Omega)\right|_{k=\lfloor\rho\rfloor}>\left.\bar{\pi}(k, w, \Omega)\right|_{k=\lceil\rho]}, \\ \lceil\rho\rceil, & \lfloor\rho\rfloor>1,\left.\bar{\pi}(k, w, \Omega)\right|_{k=\lfloor\rho\rfloor}<\left.\bar{\pi}(k, w, \Omega)\right|_{k=\lceil\rho\rceil},\end{cases}
$$

$$
\rho=\frac{1}{L} \sqrt{\frac{2 F}{h_{0} \Delta T \sum_{j=1}^{N}\left(1 / n_{j}\right) Q_{j}}}
$$$$
\sum_{m \in M} \sum_{j=1}^{N} \sum_{i=1}^{N} \delta_{i}^{r} \delta_{j}^{r} Q_{j} x_{i j}^{r m} \leq G, \quad \forall r=1,2, \ldots, k L,
$$$$
\sum_{m \in M} \sum_{j=1}^{N} x_{i j}^{r m}=1, \quad i \neq j, \forall i=1,2, \ldots, N
$$$$
\forall r=1,2, \ldots, k L
$$

$\sum_{j=1}^{N} x_{i j}^{r m} \leq 1, \quad i \neq j, \forall i=1,2, \ldots, N, \forall r=1,2, \ldots, k L$,

$$
\begin{gathered}
\sum_{j=1}^{N} x_{i j}^{r m}-\sum_{j=1}^{N} x_{j i}^{r m}=0, \quad i \neq j, \quad \forall i=1,2, \ldots, N, \\
\forall r=1,2, \ldots, k L, \forall m \in M, \\
\sum_{j=1}^{N} x_{0 j}^{r m}=1, \quad \forall r=1,2, \ldots, k L, \\
\sum_{j=1}^{N} x_{j 0}^{r m}=1, \quad \forall r=1,2, \ldots, k L, \\
x_{i j}^{r m} \in\{0,1\}, \quad i \neq j, \quad \forall i, j \in V, \forall m \in M, \\
\delta_{j}^{r}=\left\{\begin{array}{ll}
1, & r=z n_{j} \\
0, & r \neq z n_{j}
\end{array}, \quad z\right. \text { is integer, } \\
\Omega=\left\{x_{i j}^{r m} \mid i, j \in V, m \in M, r=1,2, \ldots, L\right\} .
\end{gathered}
$$

Constraints (23) to (26) relate to optimal strategy of the retailer. Constraint (26) ensures that each retailer's profit is positive. Constraints (27) to (29) relate to optimal strategy of the distributor. Constraint (30) is capacity limit of delivery vehicle. Constraints (31) to (32) limit that one retailer is visited by exactly one vehicle. Constraint (33) ensures the equation of flow between retailers. Constraints (34) and (35) guarantee that the starting point and the terminal point of every distribute route are the distribute center. Finally, restrictions (34) to (38) define the nature of the variables.

\section{Model on Regular Chain Mode}

Proposition 2. On regular chain mode, when the average profit of supply chain is maximumzied, there is no surplus products at terminal time of order cycle. That is, the relationship between any delivery cycle of retailer, $n_{j} \Delta T$, and retailer $j$ time of the inventory level decreased to zero, $t_{j}^{r}$, satisfies

$$
t_{j}^{r}=n_{j} \Delta T-\varepsilon_{j}^{r}, \quad \varepsilon_{j}^{r} \geq 0, r=1,2, \ldots, \frac{L}{n_{j}}, \forall j=1,2, \ldots, N
$$

Within one distribution cycle $L$, the sales revenue of retailer $j$ minus inventory holding cost, shortage cost, and deteriorating treatment cost, we have

$$
\begin{aligned}
\pi_{j}^{\prime}= & p \sum_{r=1}^{L / n_{j}} Q_{j}^{r}-h_{j} \sum_{r=1}^{L / n_{j}} \int_{0}^{t_{j}^{r}} I_{j}^{r}(t) d t-s_{j} B_{j} \sum_{r=1}^{L / n_{j}}\left(n_{j} \Delta T-t_{j}^{r}\right) \\
& -b \sum_{r=1}^{L / n_{j}} \int_{0}^{t_{j}^{r}} \theta I(t) d t .
\end{aligned}
$$


Then, we have the average profit of retailer $j$ :

$$
\begin{aligned}
\bar{\pi}_{j}^{\prime}= & \frac{\pi_{j}^{\prime}}{L \Delta T} \\
= & \frac{p}{L \Delta T} \sum_{r=1}^{L / n_{j}} Q_{j}^{r}-\frac{s_{j} B_{j}}{L \Delta T} \sum_{r=1}^{L / n_{j}}\left(n_{j} \Delta T-t_{j}^{r}\right) \\
& -\frac{1}{L \Delta T} \frac{B_{j}\left(h_{j}+b \theta\right)}{\theta+\alpha_{j}} \sum_{r=1}^{L / n_{j}}\left[\frac{1}{B_{j}} Q_{j}^{r}-t_{j}^{r}\right] .
\end{aligned}
$$

The distributor's order quantity, $Q$, equals the total delivery quantity in one order cycle $k L \Delta T$ :

$$
Q=\sum_{j=1}^{N} \sum_{r=1}^{k L} \delta_{j}^{r} Q_{j}^{r}=k \sum_{j=1}^{N} \sum_{r=1}^{L / n_{j}} Q_{j}^{r} .
$$

The cost of distributor is

$$
\begin{aligned}
\pi^{\prime}= & w_{0} Q+c^{\prime} Q+c^{\prime} Q \\
& +\sum_{r=1}^{k L} \sum_{m \in M} \sum_{\substack { i=0 \\
\begin{subarray}{c}{j=0 \\
j \neq i{ i = 0 \\
\begin{subarray} { c } { j = 0 \\
j \neq i } }\end{subarray}}^{N} c_{i j} \delta_{j}^{r} \delta_{i}^{r} x_{i j}^{r m}+F
\end{aligned}
$$

The partials of (43) are in sequence of purchase cost, freight of purchase products, delivery cost, inventory holding cost, and constant order cost. Then, we have the average profit of the distributor:

$$
\begin{aligned}
\bar{\pi}^{\prime}= & \frac{\pi^{\prime}}{k L \Delta T} \\
= & \frac{1}{L \Delta T}\left(w_{0}+c^{\prime}\right) \sum_{j=1}^{N} \sum_{r=1}^{L / n_{j}} Q_{j}^{r} \\
& +\frac{1}{L} h_{0} \sum_{j=1}^{N} \sum_{r=1}^{L / n_{j}}\left[(r-1) n_{j}+\frac{k-1}{2} L\right] Q_{j}^{r} \\
& +\frac{1}{L \Delta T} \sum_{r=1}^{L} \sum_{m \in M} \sum_{\substack { i=0 \\
\begin{subarray}{c}{j=0 \\
j \neq i{ i = 0 \\
\begin{subarray} { c } { j = 0 \\
j \neq i } }\end{subarray}}^{N} c_{i j} \delta_{j}^{r} \delta_{i}^{r} x_{i j}^{r m}+\frac{1}{k L \Delta T} F .
\end{aligned}
$$

From (41) and (44), we have the average profit of supply chain:

$$
\begin{aligned}
& \bar{\Pi}^{\prime}=\sum_{j=1}^{N} \bar{\pi}_{j}^{\prime}-\bar{\pi}^{\prime} \\
&=\frac{1}{L \Delta T} \sum_{j=1}^{N} \sum_{r=1}^{L / n_{j}}\left\{p-w_{0}-c^{\prime}-\frac{h_{j}+b \theta}{\theta+\alpha_{j}}-h_{0} \Delta T\right. \\
&\left.\times\left[(r-1) n_{j}+\frac{k-1}{2} L\right]\right\} Q_{j}^{r}
\end{aligned}
$$

$$
\begin{aligned}
& +\frac{1}{L \Delta T} \sum_{j=1}^{N} \sum_{r=1}^{L / n_{j}} B_{j}\left(\frac{h_{j}+b \theta}{\theta+\alpha_{j}}+s_{j}\right) t_{j}^{r} \\
& -\frac{1}{L \Delta T} \sum_{j=1}^{N} \sum_{r=1}^{L / n_{j}} s_{j} B_{j} n_{j} \Delta T \\
& -\frac{1}{L \Delta T} \sum_{r=1}^{L} \sum_{m \in M} \sum_{\substack{i=0 \\
j}}^{N} \sum_{\substack{j=0 \\
j \neq i}}^{N} c_{i j} \delta_{j}^{r} \delta_{i}^{r} x_{i j}^{r m} \\
& -\frac{1}{k L \Delta T} F .
\end{aligned}
$$

From (45), the average profit of supply chain is concave functions of $k$. So, for constant order cycle of retailers, $\Delta T L$, we can find the optimal $k^{*}, k^{*} \geq 1$, and $\bar{\Pi}^{\prime}$ approach or reach the maximum value correspondingly. Let $d \bar{\Pi}^{\prime} / d k=0$; we have

$$
k= \begin{cases}1, & \lfloor\rho\rfloor \leq 1, \\ \lfloor\rho\rfloor, & \lfloor\rho\rfloor>1, \bar{\Pi}^{\prime}\left(k^{\prime}=\lfloor\rho\rfloor\right)>\bar{\Pi}^{\prime}\left(k^{\prime}=\lceil\rho\rceil\right), \\ \lceil\rho\rceil, & \lfloor\rho\rfloor>1, \bar{\Pi}^{\prime}\left(k^{\prime}=\lfloor\rho\rfloor\right)<\bar{\Pi}^{\prime}\left(k^{\prime}=\lceil\rho\rceil\right),\end{cases}
$$

where

$$
\rho=\frac{1}{L} \sqrt{\frac{2 F}{h_{0} \Delta T \sum_{j=1}^{N} \sum_{r=1}^{L / n_{j}} Q_{j}^{r}}} .
$$

On regular mode, the optimal problem is to determine the optimal order quantity $Q$ and order cycle $k L \Delta T$, delivery quantity $Q_{j}^{r}, r=1,2, \ldots, L / n_{j}$, delivery cycle $n_{j} \Delta T$, and distribution route $\Omega$, which maxmize the average profit of supply chain. The mathematical model of inventorydistribution routing problem is

$$
\begin{gathered}
\max \bar{\Pi}^{\prime}\left(k, n_{j}, Q_{j}^{r}, \Omega\right) \\
=\max \left\{\frac { 1 } { L \Delta T } \sum _ { j = 1 } ^ { N } \sum _ { r = 1 } ^ { L / n _ { j } } \left\{p-w_{0}-c^{\prime}-\frac{h_{j}+b \theta}{\theta+\alpha_{j}}\right.\right. \\
\quad-\frac{1}{L \Delta T} \sum_{j=1}^{N} \sum_{r=1}^{L / n_{j}} B_{j}\left(\frac{h_{j}+b \theta}{\theta+\alpha_{j}}+s_{j}\right) t_{j}^{r}
\end{gathered}
$$




$$
\begin{aligned}
& -\frac{1}{L \Delta T} \sum_{j=1}^{N} \sum_{r=1}^{L / n_{j}} s_{j} B_{j} n_{j} \Delta T \\
& \left.-\frac{1}{L \Delta T} \sum_{r=1}^{L} \sum_{m \in M} \sum_{\substack { i=0 \\
\begin{subarray}{c}{j=0 \\
j \neq i{ i = 0 \\
\begin{subarray} { c } { j = 0 \\
j \neq i } }\end{subarray}}^{N} c_{i j} \delta_{j}^{r} \delta_{i}^{r} x_{i j}^{r m}-\frac{1}{k L \Delta T} F\right\},
\end{aligned}
$$

s.t.

$$
\begin{aligned}
& Q_{j}^{r}=\frac{B_{j}}{\theta+\alpha_{j}}\left[e^{\left(\theta+\alpha_{j}\right) t_{j}^{r}}-1\right], \quad \forall j=1,2, \ldots, N, \\
& n_{j} \text { is integer, } \\
& L=\left[n_{1}, n_{2}, \ldots, n_{N}\right] \text {, } \\
& k= \begin{cases}1, & \lfloor\rho\rfloor \leq 1, \\
\lfloor\rho\rfloor, & \lfloor\rho\rfloor>1, \bar{\Pi}^{\prime}\left(k^{\prime}=\lfloor\rho\rfloor\right)>\bar{\Pi}^{\prime}\left(k^{\prime}=\lceil\rho\rceil\right), \\
\lceil\rho\rceil, & \lfloor\rho\rfloor>1, \bar{\Pi}^{\prime}\left(k^{\prime}=\lfloor\rho\rfloor\right)<\bar{\Pi}^{\prime}\left(k^{\prime}=\lceil\rho\rceil\right),\end{cases} \\
& \rho=\frac{1}{L} \sqrt{\frac{2 F}{h_{0} \Delta T \sum_{j=1}^{N} \sum_{r=1}^{L / n_{j}} Q_{j}^{r}}}, \\
& \sum_{m \in M} \sum_{j=1}^{N} \sum_{i=1}^{N} \delta_{i}^{r} \delta_{j}^{r} Q_{j} x_{i j}^{r m} \leq G, \quad \forall r=1,2, \ldots, k L, \\
& \sum_{m \in M} \sum_{j=1}^{N} x_{i j}^{r m}=1, \quad i \neq j, \quad \forall i=1,2, \ldots, N, \\
& \forall r=1,2, \ldots, k L \text {, } \\
& \sum_{j=1}^{N} x_{i j}^{r m} \leq 1, \quad i \neq j, \quad \forall i=1,2, \ldots, N \\
& \forall r=1,2, \ldots, k L, \forall m \in M \\
& \sum_{j=1}^{N} x_{i j}^{r m}-\sum_{j=1}^{N} x_{j i}^{r m}=0, \quad i \neq j, \forall i=1,2, \ldots, N, \\
& \forall r=1,2, \ldots, k L, \forall m \in M, \\
& \sum_{j=1}^{N} x_{0 j}^{r m}=1, \quad \forall r=1,2, \ldots, k L, \\
& \sum_{j=1}^{N} x_{j 0}^{r m}=1, \quad \forall r=1,2, \ldots, k L, \\
& n_{j} \leq n_{\max } \\
& x_{i j}^{r m} \in\{0,1\}, \quad i \neq j, \forall i, j \in V, \forall m \in M,
\end{aligned}
$$

$$
\begin{gathered}
\delta_{j}^{r}=\left\{\begin{array}{ll}
1, & r=z n_{j} \\
0, & r \neq z n_{j}
\end{array}, \quad z\right. \text { is integer, } \\
\Omega=\left\{x_{i j}^{r m} \mid i, j \in V, m \in M, r=1,2, \ldots, L\right\} .
\end{gathered}
$$

Constraints (49) to (52) illustrate the relationship between $n_{j}$ and $Q_{j}, L, k$. Constraint (53) is capacity limit of delivery vehicle. Constraints (54) and (55) limit that one retailer is visited by exactly one vehicle. Constraint (56) ensures the equation of flow between retailers. Constraints (57) and (58) guarantee that the starting point and the terminal point of every distribute route are the distribute center. Constraint (59) is length limit of retailer's order cycle. Finally, restrictions (60) to (62) define the nature of the variables.

\section{Solution}

In the following, improved genetic algorithms are developed to solve inventory-distribution routing problem of agriculture products supply chain.

5.1. HAGA for Franchise Chain Mode. On franchise chain mode, the optimal order quantity and order cycle rely on wholesale price, so the key to solve the optimal problem is distribution routing. A heuristic adaptive genetic algorithm (HAGA) is developed for it. Heuristic rules mean that adding some routes which obtained by nearest neighbor algorithm and saving algorithm to initial population. Adaptive rules are that crossover ratio and mutation ratio vary with the population's evolution state.

Natural number code is adopted, and the chromosome structure is

$$
\begin{aligned}
& \left(0, a_{11}, a_{12}, \ldots, a_{1 u}, 0, a_{21}, a_{22}, \ldots, a_{2 v}, 0, \ldots, 0, a_{l 1},\right. \\
& \left.a_{l 2}, \ldots, a_{l w}\right),
\end{aligned}
$$

where $a_{\text {st }}$ denotes serial number of task, 0 denotes distribution center, and gene fragment between two adjacent 0 is a delivery route.

To improve quality and stability of evolution, elitist model and elitist selection strategy is adopted.

PMX operator is used in crossover operation, and reversed operator is used in mutation operation. Crossover ratio $P_{c}$ and mutation ratio $P_{m}$ are expressed as follows:

$$
P_{c}= \begin{cases}e_{c 1} \frac{J_{\mathrm{avg}}-J_{\min }}{J}, & J_{\mathrm{avg}}-J_{\min }<J, \\ e_{c 2}, & J_{\mathrm{avg}}-J_{\min } \geq J,\end{cases}
$$

where $e_{c 1}, e_{c 2}$ are constant, $0 \leq e_{c 1} \leq 1,0 \leq e_{c 2} \leq 1 . J_{\text {avg }}$ is the average fitness value of the population. $J_{\min }$ is the fitness value of the best chromosome in the population. $J$ is the better one in the cross couple

$$
P_{m}= \begin{cases}e_{m 1} \frac{J-J_{\min }}{J_{\mathrm{avg}}-J_{\min }}, & J-J_{\min }<J_{\mathrm{avg}}-J_{\min }, \\ e_{m 2}, & J-J_{\min } \geq J_{\mathrm{avg}}-J_{\min },\end{cases}
$$

where $e_{m 1}, e_{m 2}$ are constant, $0 \leq e_{m 1} \leq 1,0 \leq e_{m 2} \leq 1$. 
Take time limitation of calculating or generationsstagnancy as algorithm termination rule. The algorithm procedure is as follows.

Step 1. According to (23) to (25), determine the optimal order cycle and order quantity of the retailers. Then, determine the distribution cycle $L$ and tasks in the order interval period. Furthermore, obtain the value of $\rho$ via (29).

Step 2. If $\lfloor\rho\rfloor \leq 1$, let order cycle coefficient of the distributor $k=1$ and obtain the optimal order cycle and order quantity of the distributor. Then, call subroutine of HAGA, seeking the optimal distribution route of every delivery interval period, go to Step 4.

Step 3. If $\lfloor\rho\rfloor>1$, let $k=\lfloor\rho\rfloor$ and $k=\lceil\rho\rceil$ and calculate, respectively, the optimal order cycle and order quantity of the distributor. Then, call subroutine of HAGA, seeking the optimal distribution route of every delivery interval period; calculate the corresponding distributor's average profit. Furthermore, determine the optimal value of $k$.

The algorithm procedure of HAGA is as follows.

Step (1): yield initial population, evaluate every chromosome, and save the elitist chromosome.

Step (2): selection, crossover, and mutation operation, yield the new population. Evaluate the new population, find the elitist chromosome in the new population, and refresh the elitist chromosome saved.

Step (3): if the elitist model or the super iteration number reaches the upper limitation go to Step (5).

Step (4): go to Step (2).

Step (5): output the optimal solution.

Step 4. Output the optimal solution: include in the optimal order cycle, optimal order quantity of the distributor and retailers, and the optimal distribution routes.

5.2. Two-Layer Genetic Algorithm Based on Oddment Modification for Regular Chain Mode. On regular chain mode, the supply chain is concentrated, and it is needed to seek the optimal solution of all decision variables. On the other hand, owing to the capacity limitation and vehicle start-up costs, sometimes the delivery quantity of some retailer will be less than that have ordered to avoid the case of distribution route with little delivery quantity. Therefore, it is more difficult to solve the optimal problem on regular chain mode than that on franchise chain mode. A two-layer genetic algorithm based on oddment modification is developed to solve it. The algorithm procedure of the upper layer is as follows.

Step 1. In the upper layer, yield initial population and calculate the initial delivery quantity of every retailer according to (23).

Step 2. The upper layer transfers the initial delivery quantity of every retailer to the lower layer and obtains the modification schemes from the lower layer. Then, evaluate
TABLE 1: Parameter values of retailers.

\begin{tabular}{lccc}
\hline & $B_{j}$ & $h_{j}$ & $\alpha_{j}$ \\
\hline$j=1$ & 40 & 0.22 & 0.2 \\
$j=2$ & 100 & 0.16 & 0.15 \\
$j=3$ & 80 & 0.1 & 0.16 \\
$j=4$ & 30 & 0.18 & 0.16 \\
$j=5$ & 150 & 0.2 & 0.1 \\
$j=6$ & 100 & 0.15 & 0.2 \\
$j=7$ & 80 & 0.1 & 0.18 \\
$j=9$ & 60 & 0.2 & 0.1 \\
$j=9$ & 120 & 0.2 & 0.2 \\
$j=10$ & 90 & 0.14 & 0.1 \\
$j=11$ & 80 & 0.25 & 0.18 \\
$j=12$ & 20 & 0.1 & 0.1 \\
$j=13$ & 130 & 0.2 & 0.16 \\
$j=14$ & 70 & 0.15 & 0.2 \\
$j=15$ & 40 & 0.25 & 0.15 \\
$j=16$ & 100 & 0.18 & 0.05 \\
$j=17$ & 50 & 0.25 & 0.15 \\
$j=18$ & 110 & 0.1 & 0.1 \\
$j=19$ & 30 & 0.2 & 0.2 \\
$j=20$ & 90 & 0.2 & 0.2 \\
\hline
\end{tabular}

the modification schemes. If the current best solution is better than the maintaining optimum individual, refresh the optimum individual.

Step 3. If calculating time or generations-stagnancy reaches the upper limitation go to Step 5.

Step 4. Selection, crossover, and mutation operation yield the new population; go to Step 2 .

Step 5. Output the optimal solution.

The algorithm procedure of the lower layer is as follows.

Step (1): according to the order cycle and order quantity of the retailers transferred by the upper layer, determine the distribution cycle $L$ and delivery tasks in every order interval period by (49) to (52). Then, calculate $\rho$ by (52).

Step (2): if $\lfloor\rho\rfloor \leq 1$, let order cycle coefficient of the distributor $k=1$ and obtain the optimal order cycle and order quantity of the distributor. Then, call subroutine of HAGA, seeking the optimal distribution route of every delivery interval period; go to Step (4).

Step (3): if $\lfloor\rho\rfloor>1$, let $k=\lfloor\rho\rfloor$ and $k=\lceil\rho\rceil$, calculate, respectively, the optimal order cycle and order quantity of the distributor. Then, call subroutine of HAGA, seeking the optimal distribution route of 


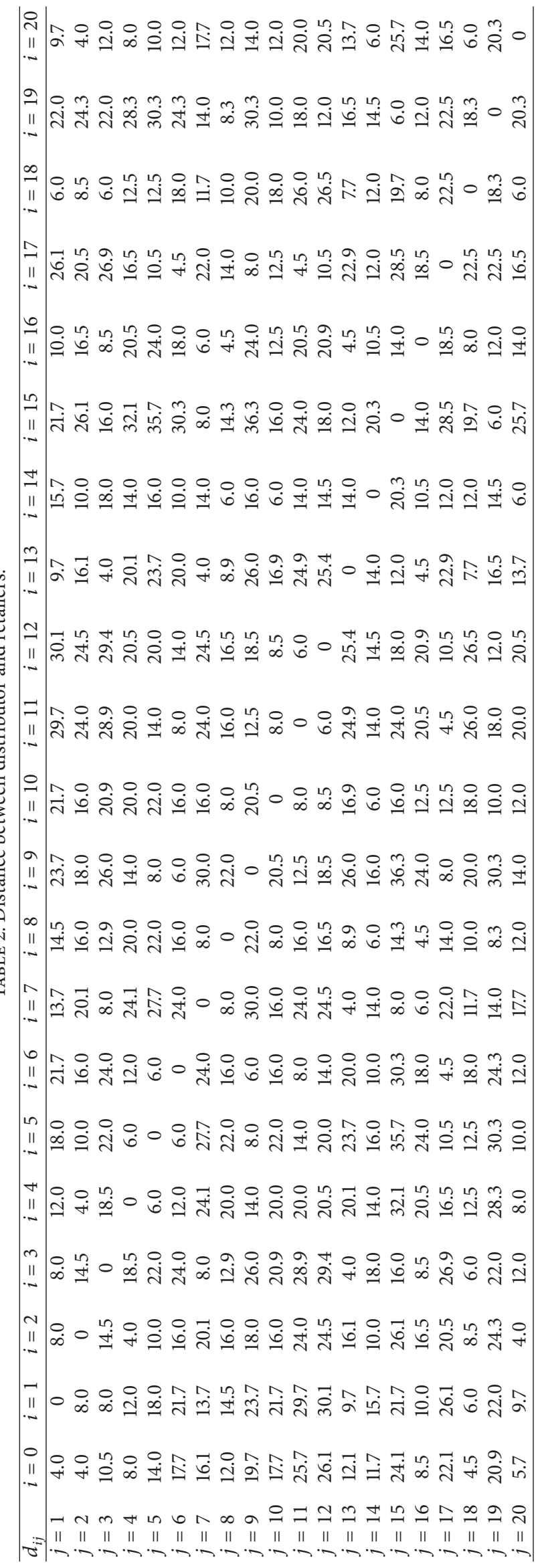


every delivery interval period, caculate the corresponding distributor's average profit. Furthermore, determine the optimal value of $k$.

Step (4): check the distribution scheme obtained by HAGA subroutine and divide the total delivery quantity in every distribution interval period by vehicle capacity $G$; if the remainders do not exceed the threshold $\Delta G$, go to Step (6).

Step (5): relaxing the vehicle capacity limitation to $G+$ $\Delta G$, call subroutine of HAGA and calculate again (put the current best distribution routes into initial population). Compare the new distribution routes with vehicle capacity and set the surplus delivery quantity as oddment. Then, by selecting retailers which have the lowest shortage cost and reducing their delivery quantity, obtain the modification delivery quantity. Furthermore, compare the optimal results after oddment modification with those of unmodified. If the new scheme is worse than the old, let the modification delivery quantity be zero.

Step (6): transfer the optimal result to the upper layer and include in the modification delivery quantity of retailers, the optimal order cycle and quantity of the distributor, and the optimal distribution routes.

\section{Numerical Study}

To illustrate the frontal model and algorithm, we consider a distribution network with one distributor and twenty retailers. Some parameter values are as follows: $\Delta T=1$, $n_{\max }=4, p=10, \theta=0.05, b=0.1, s=0.6, w=7.5$, $w_{0}=3, F_{j}=20, F_{0}=200, h_{0}=0.05, c=0.5, c=4, c_{0}=80$, and $G=1500$. Other relevant parameter values are shown in Tables 1 and 2 in the appendix.

On franchise chain mode, the parameters of HAGA algorithm are as follows: the number of initial population is $30, P_{s}=0.1, e_{c 1}=e_{c 2}=0.6, e_{m 1}=e_{m 2}=0.2$, upper limit of the elitist model is 15, and super iteration number is 150 .

In Table 3, the optimization results of order cycle length, order quantity, and average profit for every retailer are listed. Correspondingly, the optimal average profit of the retailers sums up to 3457.24 . For the distributor, the optimal order cycle length $K L^{*}=6$, the optimal order quantity $Q_{0}^{*}=$ 11533.17, and the optimal average profit is 6880.468. On franchise chain mode, optimal average profit of the supply chain is 10337.71. An optimal distribution route schedule of the distributor, in which $K L=6$, is shown in Table 4 .

On regular chain mode, the parameters of the upper layer algorithm are as follows: the number of initial population is $40, P_{s}=0.1, P_{c}=0.8, P_{m}=0.2$, and upper limit of the elitist model is 20 . The lower layer algorithm has the same parameters as those of algorithm on franchise chain mode and the oddment modification threshold $\Delta G=100$.

In Table 5, the optimization results of order cycle length and order quantity for every retailer are listed. Correspondingly, for the distributor, the optimal order cycle length $K L^{*}=2$ and the optimal order quantity $Q_{0}^{*}=3543.11$. On regular chain mode, optimal average profit of the supply
TABLE 3: Optimal results of retailers on ranchise chain mode.

\begin{tabular}{lccc}
\hline & $n_{j}$ & $Q_{j}$ & $\pi_{j}$ \\
\hline$j=1$ & 3 & 178.72 & 85.50 \\
$j=2$ & 1 & 110.70 & 221.17 \\
$j=3$ & 2 & 198.84 & 182.60 \\
$j=4$ & 2 & 74.56 & 59.45 \\
$j=5$ & 1 & 161.83 & 328.96 \\
$j=6$ & 3 & 446.80 & 237.46 \\
$j=7$ & 3 & 345.64 & 188.74 \\
$j=9$ & 1 & 64.73 & 119.59 \\
$j=9$ & 2 & 311.38 & 278.58 \\
$j=10$ & 1 & 97.10 & 192.22 \\
$j=11$ & 2 & 203.16 & 173.11 \\
$j=12$ & 3 & 75.77 & 35.27 \\
$j=13$ & 1 & 144.66 & 292.43 \\
$j=14$ & 3 & 312.76 & 164.22 \\
$j=15$ & 2 & 98.36 & 78.29 \\
$j=16$ & 1 & 105.17 & 207.51 \\
$j=17$ & 2 & 122.96 & 100.37 \\
$j=18$ & 1 & 118.68 & 241.69 \\
$j=19$ & 3 & 134.04 & 63.63 \\
$j=20$ & 2 & 233.54 & 206.43 \\
\hline
\end{tabular}

TABle 4: Optimal distribution route of a distribution cycle on ranchise chain mode.

\begin{tabular}{lc}
\hline $\begin{array}{l}\text { Serial number } \\
\text { of distribution time }\end{array}$ & $\Omega$ \\
\hline$n=1$ & $0-2-5-10-8-16-13-18-0$ \\
$n=2$ & $0-2-4-5-9-17-11-10-20-0$, \\
$n=3$ & $0-18-16-8-15-13-3-0$ \\
$n=4$ & $0-2-5-6-12-14-0,0-1-18-13-7-16-8-19-10-0$ \\
$n=5$ & $0-2-4-5-9-17-11-10-20-0$, \\
$n=6$ & $0-18-16-8-15-13-3-0$ \\
& $0-2-5-10-8-16-13-18-0$ \\
\end{tabular}

chain is 11290.3. An optimal distribution route schedule of the distributor, in which $K L=2$, is shown in Table 6 .

To illustrate the validity of oddment modification, we let the vehicle capacity to be 1000 , then use the two-layer genetic algorithm based on oddment modification and without oddment modification, respectively, to calculate the problem of regular chain mode. The optimization results are shown in Table 7, in which oddment modification is done when $n=1$; two routes are combined, and the corresponding modification delivery quantity is 85 .

From Table 7, when the tasks of some routes are not adequate, by reducing the number of distribution routes, oddment modification may save more transportation cost at the cost of lesser shortage. It contributes to improvement of the supply chain's total profit. 
TABLE 5: Optimal results of retailers on regular chain mode.

\begin{tabular}{llc}
\hline & $n_{j}$ & $Q_{j}$ \\
\hline$j=1$ & 2 & 103.79 \\
$j=2$ & 1 & 110.70 \\
$j=3$ & 2 & 198.84 \\
$j=4$ & 2 & 74.57 \\
$j=5$ & 1 & 161.83 \\
$j=6$ & 2 & 259.49 \\
$j=7$ & 2 & 203.16 \\
$j=9$ & 1 & 64.73 \\
$j=9$ & 1 & 136.33 \\
$j=10$ & 1 & 97.10 \\
$j=11$ & 1 & 89.95 \\
$j=12$ & 2 & 46.65 \\
$j=13$ & 1 & 144.66 \\
$j=14$ & 2 & 181.64 \\
$j=15$ & 2 & 98.36 \\
$j=16$ & 2 & 105.17 \\
$j=17$ & 1 & 55.35 \\
$j=18$ & 1 & 118.68 \\
$j=19$ & 1 & 77.85 \\
$j=20$ & 2 & 233.54 \\
\hline
\end{tabular}

TABLE 6: Optimal distribution route of a distribution cycle on regular chain mode.

\begin{tabular}{lc}
\hline $\begin{array}{l}\text { Serial number of } \\
\text { distribution time }\end{array}$ & $\Omega$ \\
\hline$n=1$ & $0-2-5-9-17-11-10-8-16-13-18-0$ \\
$n=2$ & $0-2-4-5-9-6-17-11-12-10-14-20-0$, \\
& $0-1-3-13-7-15-19-8-16-18-0$ \\
\hline
\end{tabular}

TABLE 7: $G=1000$, optimal routes of a distribution cycle on regular chain mode.

\begin{tabular}{lll}
\hline & $\begin{array}{l}\text { Without oddment } \\
\text { modification }\end{array}$ & $\begin{array}{l}\text { With oddment } \\
\text { modification }\end{array}$ \\
\hline$\Omega$ & & \\
$n=1$ & $0-2-5-9-17-11-10-0$, & $0-2-5-9-17-11-10-8-16-$ \\
& $0-8-16-13-18-0$ & $13-18-0$ \\
& & \\
$n=2$ & $0-2-4-5-9-6-17-11-12-$ & $0-2-4-5-9-6-17-11-12-$ \\
& 0, & 0, \\
& $0-1-3-13-7-15-19-16-18-$ & $0-1-3-13-7-15-19-16-18-$ \\
& 0, & 0, \\
\hline $\begin{array}{l}\text { Average profit of } \\
\text { supply chain }\end{array}$ & $0-20-14-10-8-0$ & $0-20-14-10-8-0$ \\
\hline
\end{tabular}

For supply chain, the character of centralized control is better than that of decentralized control. In the frontal case, the average profit of supply chain on regular chain mode, 11290.3 , is higher than that on franchise chain mode, 10337.7. However, a fixed order cost, $F_{0}=20$, is considered for
TABLE 8: Profit of supply chain with different wholesale price, on ranchise chain mode.

\begin{tabular}{lccc}
\hline Wholesale price & \multicolumn{3}{c}{ Average profit } \\
& Retailers & Distributor & Supply chain \\
\hline$w=7.48$ & 3495.89 & 6717.02 & 10212.91 \\
$w=7.49$ & 3476.48 & 6900.75 & 10377.23 \\
$w=7.50$ & 3457.24 & 6880.47 & 10337.71 \\
$w=7.54$ & 3380.40 & 6927.29 & 10307.69 \\
$w=7.60$ & 3266.69 & 6898.71 & 10165.40 \\
$w=8.00$ & 2534.11 & 7419.28 & 9953.39 \\
$w=8.99$ & 791.48 & 8794.95 & 9586.43 \\
\hline
\end{tabular}

every retailer on franchise chain mode, while no order cost is considered on regular chain mode. Further, we remove the retailers' fixed order cost on franchise chain, that is, let $F_{0}=0$. Owing to no transportation cost, shorter order cycle means less cost, so the retailers' optimal length of order cycle is 1 . Hence the distributor's optimal length of order cycle and distribution cycle will be 1 . The corresponding optimal distribution routes are 0-2-4-5-9-6-17-11-12-10-14-20-0 and $0-1-3-13-7-15-19-8-16-18-0$, the distributor's optimal average profit is 6587.65, and the optimal average profit of supply chain is 10361.03, which is higher than that with $F_{0}=20$.

Table 8 shows the average profit with different wholesale price on franchise chain mode. From Table 8, while wholesale price contract cannot coordinate the supply chain on franchise chain mode, profit distribution can be regulated by changing the wholesale price. For retailers, higher wholesale price means less profit. For the distributor, the opposite tendency is true in most cases, but owing to transportation cost, the profit on $w=7.50$ is less than that of $w=7.49$. The average profit of supply chain reaches the maximum (10377.23) at $w=7.49$, which is less than that on regular chain mode.

\section{Conclusions}

We have discussed the inventory-distribution routing problem of agriculture products supply chain with one distributor and multiple retailers, based on franchise chain mode and regular chain mode. The corresponding mathematical models are presented.

For the franchise chain model, the optimal order cycle and order quantity of the retailers are dependent on wholesale price; therefore the key to solve the optimal problem is to solve the static multiperiod distribution routing problem. Then, an heuristic adaptive genetic algorithm is proposed for the model of franchise chain. For the regular chain model, a two-layer genetic algorithm based on oddment modification is proposed, which searches for the optimal distribution routes, the optimal order cycle, and order quantity for both the retailers and the distributor. The upper layer provides the distribution cycle and delivery quantity for the retailers, and the lower layer seeks the optimal order cycle, order quantity, distribution routes, and the rational oddment modification 
number for the distributor. The validity of the algorithms is also demonstrated by numerical examples.

The supply chain on regular chain mode is centralized controlling, and its average profit is higher than that on franchise chain mode, which is decentralized controlling. On franchise chain mode, profit distribution between the retailers and the distributor can be regulated by changing the wholesale price.

\section{Appendix}

For more details see Table 2.

\section{References}

[1] A. Federgruen and P. Zipkin, "A combined vehicle routing and inventory allocation problem," Operations Research, vol. 32, no. 5, pp. 1019-1037, 1984.

[2] L. D. Burns, R. W. Hall, D. E. Blumenfeld, and C. F. Daganzo, "Distribution strategies that minimize transportation and inventory costs," Operations Research, vol. 33, no. 3, pp. 469490, 1985.

[3] C. F. Daganzo, "A comparison of in-vehicle and out-of- vehicle freight consolidation strategies," Transportation Research B, vol. 22, no. 3, pp. 173-180, 1988.

[4] S. Anily and A. Federgruen, "One warehouse multiple retailer systems with vehicle routing costs," Management Science, vol. 36, no. 1, pp. 92-114, 1990.

[5] P. Trudeau and M. Dror, "Stochastic inventory routing: route design with stockouts and route failures," Transportation Science, vol. 26, no. 3, pp. 171-184, 1992.

[6] R. Ernst and D. F. Pyke, "Optimal base stock policies and truck capacity in a two-echelon system," Naval Research Logistics, vol. 40, no. 7, pp. 879-903, 1993.

[7] Y. Herer and R. Roundy, "Heuristics for a one-warehouse multiretailer distribution problem with performance bounds," Operations Research, vol. 45, no. 1, pp. 102-115, 1997.

[8] S. Viswanathan and K. Mathur, "Integrating routing and inventory decisions in one-warehouse multiretailer multiproduct distribution systems," Management Science, vol. 43, no. 3, pp. 294-312, 1997.

[9] L. Bertazzi, M. G. Speranza, and W. Ukovich, "Minimization of logistic costs with given frequencies," Transportation Research B, vol. 31, no. 4, pp. 327-340, 1997.

[10] F. Chen and Y. S. Zheng, "Near-optimal echelon-stock (R, nQ) policies in multistage serial systems," Operations Research, vol. 46, no. 4, pp. 592-602, 1998.

[11] L. Bertazzi and M. G. Speranza, "Continuous and discrete shipping strategies for the single link problem," Transportation Science, vol. 36, no. 3, pp. 314-325, 2002.

[12] L. Wang, S. R. Sun, and X. C. Wu, "Distribution strategies that minimize inventory and vehicle routing costs in a two-echelon distribution system," System Engineering Theory \& Practice, vol. 25, no. 10, pp. 33-38, 2005.

[13] D. Zhao, L. Jun, and M. Danxiang, "An algorithm for stochastic demand inventory routing problem," System Engineering, vol. 24, no. 5, pp. 23-28, 2006.

[14] G. Padmanabhan and P. Vrat, "EOQ models for perishable items under stock dependent selling rate," European Journal of Operational Research, vol. 86, no. 2, pp. 281-292, 1995. 


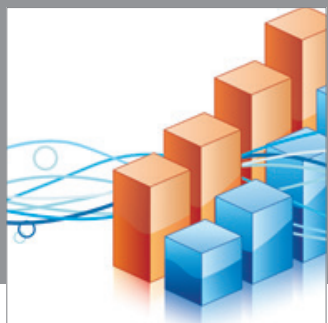

Advances in

Operations Research

mansans

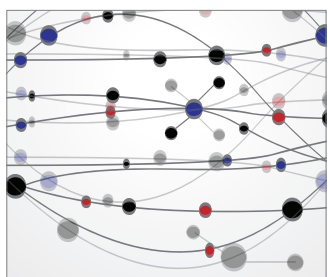

The Scientific World Journal
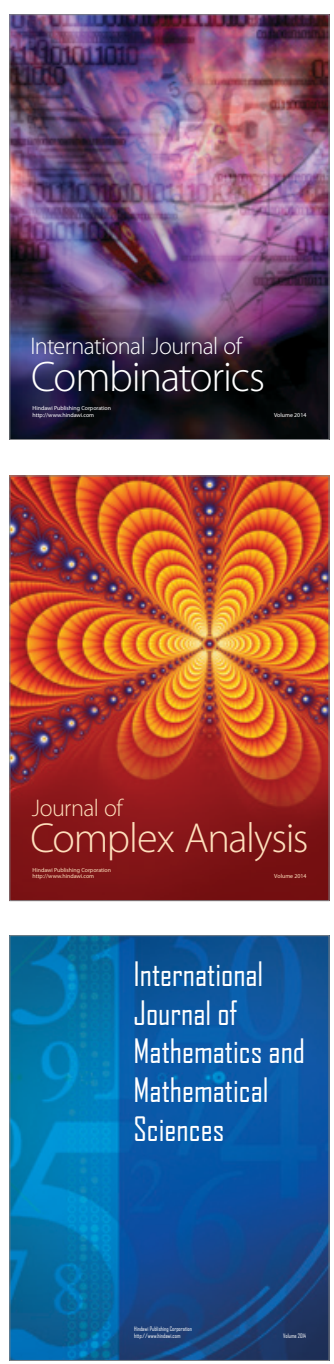
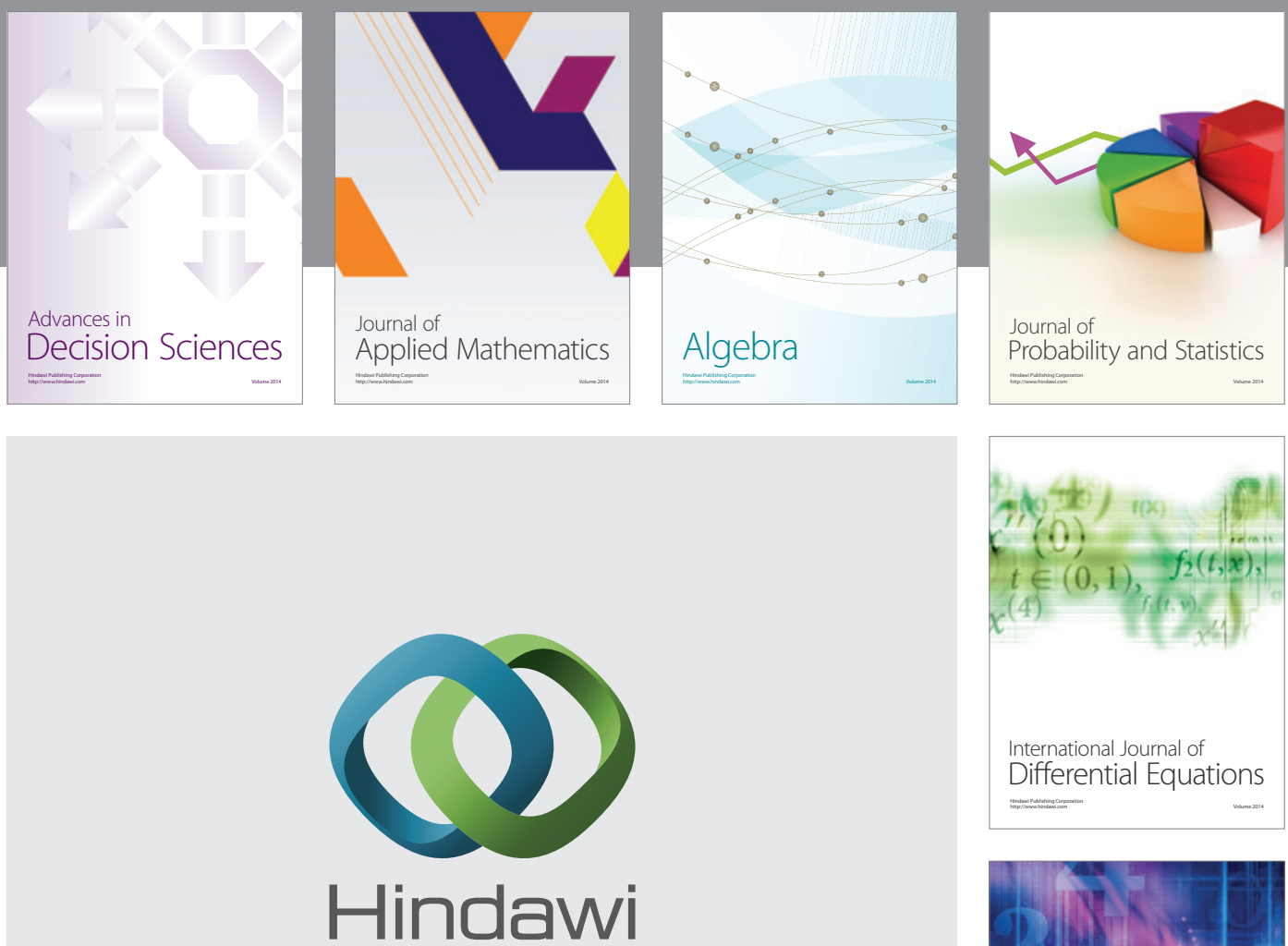

Submit your manuscripts at http://www.hindawi.com
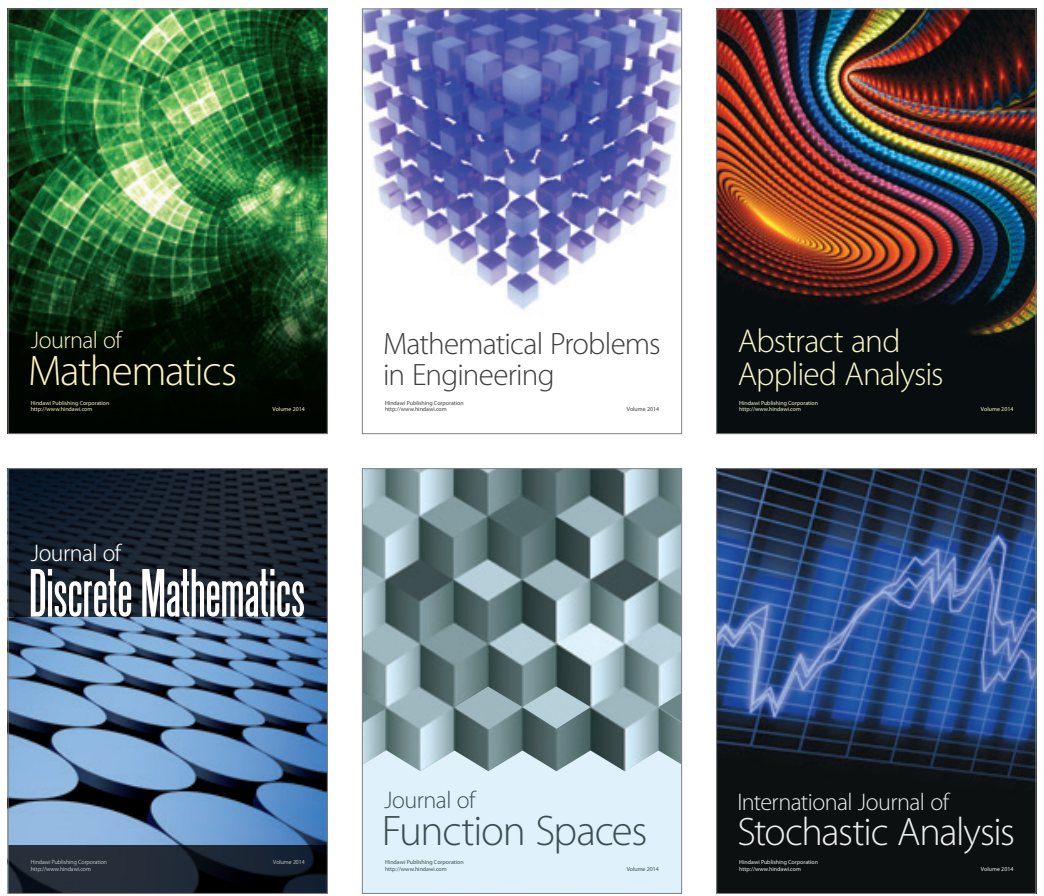

Journal of

Function Spaces

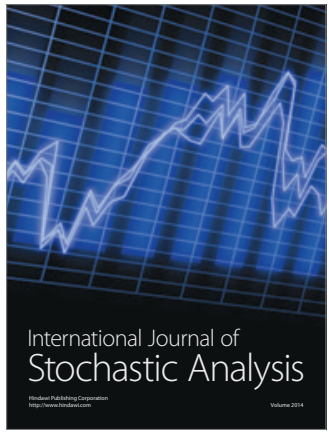

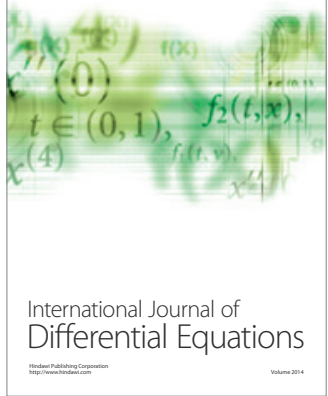
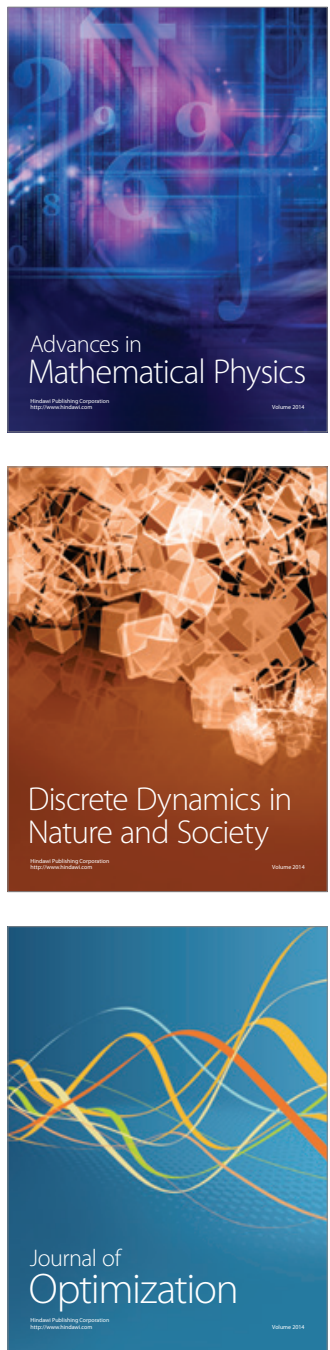